\title{
A numerical method for nonlinear eigenvalue problems using contour integrals
}

\author{
Junko Asakura $^{1}$, Tetsuya Sakurai ${ }^{2}$, Hiroto Tadano ${ }^{2}$, Tsutomu Ikegami ${ }^{3}$ and Kinji Kimura ${ }^{4}$ \\ Research and Development Division, Square Enix Co. Ltd., Shinjuku Bunka Quint Bldg. 3- \\ 22-7 Yoyogi, Shibuya-ku, Tokyo 151-8544, Japan ${ }^{1}$ \\ Department of Computer Science, University of Tsukuba, 1-1-1 Tennoudai, Tsukuba, Ibaraki \\ 305-8573, Japan $^{2}$ \\ Information Technology Research Institute, AIST, 1-1-1 Umezono, Tsukuba, Ibaraki 305-8568, \\ $\mathrm{Japan}^{3}$ \\ Graduate School of Informatics, Kyoto University, Yoshida-honmachi, Sakyo-ku, Kyoto, 606- \\ 8501, $\operatorname{Japan}^{4}$ \\ E-mailsakurai@cs.tsukuba.ac.jp
}

Received February 14, 2009, Accepted May 1, 2009

\begin{abstract}
A contour integral method is proposed to solve nonlinear eigenvalue problems numerically. The target equation is $F(\lambda) \boldsymbol{x}=0$, where the matrix $F(\lambda)$ is an analytic matrix function of $\lambda$. The method can extract only the eigenvalues $\lambda$ in a domain defined by the integral path, by reducing the original problem to a linear eigenvalue problem that has identical eigenvalues in the domain. Theoretical aspects of the method are discussed, and we illustrate how to apply of the method with some numerical examples.
\end{abstract}

Keywords nonlinear eigenvalue problem, contour integral, analytic matrix function

Research Activity Group Algorithms for Matrix / Eigenvalue Problems and their Applications

\section{Introduction}

We consider a numerical method using contour integrals to solve nonlinear eigenvalue problems. The nonlinear eigenvalue problem (NEP) involves finding eigenpairs $(\lambda, \boldsymbol{x})$ that satisfy $F(\lambda) \boldsymbol{x}=\mathbf{0}$, where the matrix $F(\lambda)$ is an analytic matrix function of $\lambda$. NEPs appear in a variety of problems in science and engineering, such as accelerator designs [1] and delay differential equations [2].

We herein propose a numerical method using contour integrals to solve eigenvalue problems for analytic matrix functions. The method is closely related to the SakuraiSugiura (SS) method for generalized eigenvalue problems [3], and inherits many of its strong points including suitability for execution on modern distributed parallel computers. We have already extended the SS method to polynomial eigenvalue problems [4]. In this paper, we will further generalize the SS method to eigenvalue problems of analytic matrix functions. In the SS method, the original problem is converted to a generalized eigenvalue problem whose dimension is smaller than the original one. The converted problem is obtained numerically by solving a set of linear equations. These linear equations are derived from the original problem and can form a large system, but they are independent and can be solved in parallel. Moreover, the proposed method is free from the fixed point iterations required in Newton's method. In this paper, the extension of the SS method for NEPs is discussed from a theoretical point of view. Some numerical examples are also reported, with results that are consistent with the theory.
The reminder of the present paper is organized as follows. In the next section, we introduce the Smith form for analytic matrix functions, which is a natural extension of the Smith form for matrix polynomials [5]. In Section 3, we present the numerical method for solving NEPs by means of the SS method and discuss theoretical results related to the proposed method. In Section 4 , we present the algorithm of the SS method for the case where the integral path is given by a circle and numerical integration is performed using the trapezoidal rule. Some numerical examples are shown in Section 5. Finally, conclusions and suggestions for future research are presented in Section 6.

\section{Canonical form for matrix analytic functions}

Let $F(z)$ be an analytic matrix function defined in a simply connected region in $\mathbb{C}$. The matrix $F(z)$ is called regular if the determinant of $F(z)$ is not identically zero in a domain $\Omega$.

We introduce the Smith form for analytic matrix functions [5].

Theorem 1 Let $F(z)$ be an $n \times n$ regular matrix analytic function. Then, $F(z)$ admits the representation

$$
P(z) F(z) Q(z)=D(z),
$$

where $D=\operatorname{diag}\left(d_{1}(z), \ldots, d_{n}(z)\right)$ is a diagonal matrix of analytic functions $d_{j}(z)$ for $j=1,2, \ldots, n$ and such that $d_{j}(z) / d_{j-1}(z)$ are analytic functions for $j=2,3, \ldots, n$. In addition, $P(z)$ and $Q(z)$ are $n \times n$ regular analytic 
matrix functions with constant nonzero determinants.

The eigenpairs of the NEP are formally derived from the Smith form. Let $\boldsymbol{q}_{j}(z)$ be the column vectors of $Q(z)$ :

$$
Q(z)=\left(\boldsymbol{q}_{1}(z) \ldots \boldsymbol{q}_{n}(z)\right)
$$

and $\boldsymbol{p}_{j}(z)$ be

$$
P(z)^{\mathrm{H}}=\left(\boldsymbol{p}_{1}(z) \ldots \boldsymbol{p}_{n}(z)\right) .
$$

Let $\lambda_{1}, \ldots, \lambda_{s}$ be distinct zeros of $d_{n}(z)$ in $\Omega$. Because $d_{j}(z) / d_{j-1}(z)$ is an analytic function, $d_{j}(z)$ can be represented in terms of $\lambda_{i}$ as

$$
d_{j}(z)=h_{j}(z) \cdot \prod_{i=1}^{s}\left(z-\lambda_{i}\right)^{\alpha_{j i}}, \quad j=1,2, \ldots, n,
$$

where $h_{j}(z)$ are analytic functions and $h_{j}(z) \neq 0$ for $z \in \Omega$. In addition, $\alpha_{j i} \in \mathbb{Z}^{+}$(non-negative integer) and $\alpha_{j i} \leq \alpha_{j^{\prime} i}$ for $j<j^{\prime}$.

The eigenpairs of the NEP are related to the $\lambda_{i}$ and the $\boldsymbol{q}_{j}\left(\lambda_{i}\right)$ above as follows.

Lemma 2 Let $\boldsymbol{q}_{j}(z)$ be the vector in (2), and $\lambda_{i}$ be a zero of $d_{j}(z)$. Then, the eigenpair $\left(\lambda_{i}, \boldsymbol{q}_{j}\left(\lambda_{i}\right)\right)$ is a solution for the NEP $F(\lambda) \boldsymbol{x}=\mathbf{0}$.

Proof Because $P(z)$ and $Q(z)$ are invertible,

$$
\begin{aligned}
F\left(\lambda_{i}\right) \boldsymbol{q}_{j}\left(\lambda_{i}\right) & =P\left(\lambda_{i}\right)^{-1} D\left(\lambda_{i}\right) Q\left(\lambda_{i}\right)^{-1}\left(Q\left(\lambda_{i}\right) \boldsymbol{e}_{j}\right) \\
& =d_{j}\left(\lambda_{i}\right) P\left(\lambda_{i}\right)^{-1} \boldsymbol{e}_{j} .
\end{aligned}
$$

Since $d_{j}\left(\lambda_{i}\right)=0$, we have the result of the lemma.

(QED)

Note that if the eigenvalue $\lambda_{i}$ is simple and not degenerate, i.e., $\lambda_{i}$ is a simple zero of $\operatorname{det} F(z)$, we have $\alpha_{j i}=0$ for $j \neq n$ and $\alpha_{n i}=1$.

\section{An eigensolver using contour integrals}

In this section, we propose a numerical method using contour integrals for eigenvalue problems of analytic matrix functions.

Let $F(z)$ be an $n \times n$ regular analytic matrix function. For nonzero vectors $\boldsymbol{u}$ and $\boldsymbol{v} \in \mathbb{C}^{n}$, we define

$$
f(z):=\boldsymbol{u}^{\mathrm{H}} F(z)^{-1} \boldsymbol{v}
$$

for $z \in \Omega$ such that $|F(z)| \neq 0$, namely $d_{n}(z) \neq 0$. The existence of the Smith form allows us to prove the following theorem.

Theorem 3 Let $D(z)=\operatorname{diag}\left(d_{1}(z), \ldots, d_{n}(z)\right)$ be the Smith form for $F(z)$, and let $P(z)$ and $Q(z)$ be defined by (1). Then, $f(z)$ admits the representation

$$
f(z)=\sum_{j=1}^{n} \frac{\chi_{j}(z)}{d_{j}(z)}
$$

where $\chi_{j}(z)$ are analytic functions in $\Omega$.

Proof By Theorem 1, we obtain

$$
\begin{aligned}
f(z) & =\boldsymbol{u}^{\mathrm{H}} Q(z) D(z)^{-1} P(z) \boldsymbol{v} \\
& =\sum_{j=1}^{n} \frac{\boldsymbol{u}^{\mathrm{H}} \boldsymbol{q}_{j}(z) \boldsymbol{p}_{j}(z)^{\mathrm{H}} \boldsymbol{v}}{d_{j}(z)}
\end{aligned}
$$

$$
=\sum_{j=1}^{n} \frac{\chi_{j}(z)}{d_{j}(z)}
$$

where $\chi_{j}(z):=\boldsymbol{u}^{\mathrm{H}} \boldsymbol{q}_{j}(z) \boldsymbol{p}_{j}(z)^{\mathrm{H}} \boldsymbol{v}$.

(QED)

Let $\Gamma$ be a positively oriented closed Jordan curve in $\Omega$. Without loss of generality, we may assume that $\lambda_{1}, \ldots, \lambda_{m}(m \leq s)$ are distinct eigenvalues in the interior of $\Gamma \subset \Omega$. Assume that these eigenvalues are simple and not degenerate. Then we can suppose that $\alpha_{j i}=0$ for $j \neq n$ and $\alpha_{n i}=1$.

Definition 4 For a non-negative integer $k$, the moment $\mu_{k}$ is defined as

$$
\mu_{k}:=\frac{1}{2 \pi \mathrm{i}} \int_{\Gamma} z^{k} f(z) \mathrm{d} z, \quad k=0,1, \ldots
$$

Definition 5 Two $m \times m$ Hankel matrices $H_{m}^{<}$and $H_{m}$ can be defined as

$$
H_{m}:=\left[\mu_{i+j-2}\right]_{i, j=1}^{m}, \quad H_{m}^{<}:=\left[\mu_{i+j-1}\right]_{i, j=1}^{m} .
$$

The following theorem is one of the main results of the present paper.

Theorem 6 If $\chi_{n}\left(\lambda_{l}\right) \neq 0$ for $1 \leq l \leq m$, then the eigenvalues of the pencil $H_{m}^{<}-\lambda H_{m}$ are given by $\lambda_{1}, \ldots, \lambda_{m}$.

Proof By Theorem 3 and (5), we obtain

$$
\begin{aligned}
\mu_{k} & =\frac{1}{2 \pi \mathrm{i}} \int_{\Gamma} z^{k} f(z) \mathrm{d} z \\
& =\sum_{j=1}^{n} \frac{1}{2 \pi \mathrm{i}} \int_{\Gamma} \frac{\chi_{j}(z)}{d_{j}(z)} z^{k} \mathrm{~d} z \\
& =\sum_{l=1}^{m} \nu_{l} \lambda_{l}^{k},
\end{aligned}
$$

where $\nu_{l}:=\chi_{n}\left(\lambda_{l}\right) / d_{n}^{\prime}\left(\lambda_{l}\right)$.

Let $V_{m}$ be the Vandermonde matrix

$$
V_{m}:=\left(\begin{array}{cccc}
1 & 1 & \cdots & 1 \\
\lambda_{1} & \lambda_{2} & \cdots & \lambda_{m} \\
\vdots & \vdots & & \vdots \\
\lambda_{1}^{m-1} & \lambda_{2}^{m-1} & \ldots & \lambda_{m}^{m-1}
\end{array}\right) .
$$

Let $D_{m}:=\operatorname{diag}\left(\nu_{1}, \ldots, \nu_{m}\right), \Lambda_{m}:=\operatorname{diag}\left(\lambda_{1}, \ldots, \lambda_{m}\right)$. One can easily verify that

$$
H_{m}^{<}-\lambda_{l} H_{m}=V_{m} D_{m}\left(\Lambda_{m}-\lambda_{l} I\right) V_{m}^{\mathrm{T}} .
$$

If $\chi_{n}\left(\lambda_{l}\right) \neq 0$ for $1 \leq l \leq m$, then $\nu_{l} \neq 0$ for $1 \leq l \leq m$. Therefore, $\lambda_{1}, \ldots, \lambda_{m}$ are the eigenvalues of the pencil $H_{m}^{<}-\lambda H_{m}$.

(QED)

Therefore, we can obtain eigenvalues $\lambda_{1}, \ldots, \lambda_{m}$ of the analytic matrix function $F(z)$ by solving the generalized eigenvalue problem $H_{m}^{<} \boldsymbol{w}=\lambda H_{m} \boldsymbol{w}$. The proof of the above theorem for generalized eigenvalue problems is given in [3].

Now, we evaluate eigenvectors. Let

$$
\boldsymbol{s}_{k}:=\frac{1}{2 \pi \mathrm{i}} \int_{\Gamma} z^{k} F(z)^{-1} \boldsymbol{v} \mathrm{d} z, \quad k=0,1, \ldots, m-1,
$$


and let $S:=\left(s_{0} \ldots s_{m-1}\right)$. We obtain the following relationship between $S$ and $\boldsymbol{q}_{n}(z)$ of (2).

Lemma 7 Let $\boldsymbol{q}_{n}(z)$ be the vector in (2) and let $\left(\lambda_{l}, \boldsymbol{w}_{l}\right)$ $(1 \leq l \leq m)$ be the eigenpairs of the pencil $H_{m}^{<}-\lambda H_{m}$. Then,

$$
\boldsymbol{q}_{n}\left(\lambda_{l}\right)=c_{l} S \boldsymbol{w}_{l}, \quad c_{l} \in \mathbb{C} \backslash\{0\}
$$

for $l=1,2, \ldots, m$.

Proof From (6), we have

$$
\mathbf{0}=\left(H_{m}^{<}-\lambda_{l} H_{m}\right) \boldsymbol{w}_{l}=V_{m} D_{m}\left(\Lambda_{m}-\lambda_{l} I\right) V_{m}^{\mathrm{T}} \boldsymbol{w}_{l} .
$$

Since $V_{m}$ and $D_{m}$ are nonsingular, and $\Lambda_{m} \boldsymbol{e}_{l}=\lambda_{l} \boldsymbol{e}_{l}$, $V_{m}^{\mathrm{T}} \boldsymbol{w}_{l}$ admits the following representation:

$$
V_{m}^{\mathrm{T}} \boldsymbol{w}_{l}=\alpha_{l} \boldsymbol{e}_{l}, \quad \alpha_{l} \in \mathbb{C} \backslash\{0\} .
$$

Here, $\boldsymbol{e}_{l}$ is the $l$ th unit vector. Let $\boldsymbol{p}_{n}(z)$ be the vector in (3) and let

$$
\beta_{l}:=\frac{\boldsymbol{p}_{n}\left(\lambda_{l}\right)^{\mathrm{H}} \boldsymbol{v}}{d_{n}^{\prime}\left(\lambda_{l}\right)}
$$

for $l=1, \ldots, m$. Note that $\beta_{l} \neq 0$ if $\chi_{n}\left(\lambda_{l}\right) \neq 0$. As in the proof of Theorem 3.4, we can derive the following equation.

$$
S=\left(\boldsymbol{s}_{0} \ldots \boldsymbol{s}_{m-1}\right)=\left(\beta_{1} \boldsymbol{q}_{n}\left(\lambda_{1}\right) \ldots \beta_{m} \boldsymbol{q}_{n}\left(\lambda_{m}\right)\right) V_{m}^{\mathrm{T}} .
$$

Therefore,

$$
\boldsymbol{q}_{n}\left(\lambda_{l}\right)=\frac{1}{\beta_{l}} S V_{m}^{-\mathrm{T}} \boldsymbol{e}_{l}=\frac{1}{\beta_{l}} S \frac{1}{\alpha_{l}} \boldsymbol{w}_{l}=c_{l} S \boldsymbol{w}_{l},
$$

with $c_{l}=1 /\left(\alpha_{l} \beta_{l}\right)$ for $l=1,2, \ldots, m$.

(QED)

From Lemma 2 and Lemma 7, we have the following theorem.

Theorem 8 Let $\left(\lambda_{j}, \boldsymbol{w}_{j}\right)(j=1, \ldots, m)$ be the eigenpairs of the pencil $H_{m}^{<}-\lambda H_{m}$. Then, $\left(\lambda_{j}, \boldsymbol{x}_{j}\right)(j=$ $1, \ldots, m)$ are the eigenpairs for the NEP $F(\lambda) \boldsymbol{x}=\mathbf{0}$, where

$$
\boldsymbol{x}_{j}=S \boldsymbol{w}_{j}, \quad j=1, \ldots, m .
$$

\section{A case where $\Gamma$ is given by a circle}

Let $\Gamma=\gamma+\rho \mathrm{e}^{\mathrm{i} \theta}(0 \leq \theta<2 \pi)$ be a circle in $\Omega$ with center $\gamma$ and radius $\rho$. To retain numerical accuracy, we use the shifted and scaled moments

$$
\mu_{k}:=\frac{1}{2 \pi \mathrm{i}} \int_{\Gamma}\left(\frac{z-\gamma}{\rho}\right)^{k} f(z) \mathrm{d} z, \quad k=0,1, \ldots
$$

instead of (5). We evaluated the integral using the $N$ point trapezoidal rule, leading to the approximations for $\mu_{k}$,

$$
\mu_{k} \approx \hat{\mu}_{k}:=\frac{1}{N} \sum_{j=0}^{N-1}\left(\frac{\omega_{j}-\gamma}{\rho}\right)^{k+1} f\left(\omega_{j}\right)
$$

where $\omega_{j}=\gamma+\rho \mathrm{e}^{2 \pi \mathrm{i}(j+1 / 2) / N}$ for $j=0,1, \ldots, N-1$. Note that due to the shift and scaling, the eigenvalues $\lambda_{l}(l=1, \ldots, m)$ are also shifted and scaled. The eigenvalues of the original NEP can be recovered from $\gamma+\rho \lambda_{l}$.

The block version of the SS method for generalized eigenvalue problems was proposed in [6]. The numerical examples in [6] indicate that the block SS method has the potential to achieve greater accuracy.

Let $U$ and $V$ be $n \times L$ matrices, the column vectors of which are linearly independent. The block SS method is defined by replacing $f(z)$ in (5) with the matrix $U^{H} F(z)^{-1} V$. Accordingly, the $k$ th moment $\mu_{k}$ in (5), the Hankel matrices $H_{m}, H_{m}^{<}$, the vector $s_{k}$ in (7) and the matrix $S=\left(s_{0} \ldots s_{m-1}\right)$ are replaced by the corresponding block versions:

$$
\begin{aligned}
& M_{k}:=\frac{1}{2 \pi \mathrm{i}} \int_{\Gamma} z^{k} U^{H} F(z)^{-1} V \mathrm{~d} z, \quad k=0,1, \ldots, \\
& H_{\tilde{m} L}:=\left[M_{i+j-2}\right]_{i, j=1}^{\tilde{m}}, \quad H_{\tilde{m} L}^{<}:=\left[M_{i+j-1}\right]_{i, j=1}^{\tilde{m}}, \\
& S_{k}:=\frac{1}{2 \pi \mathrm{i}} \int_{\Gamma} z^{k} F(z)^{-1} V \mathrm{~d} z, \quad k=0,1, \ldots,
\end{aligned}
$$

and $S=\left(S_{0} \ldots S_{\tilde{m}-1}\right)$, respectively. Here $\tilde{m}$ is a positive integer such that $\tilde{m} L \geq m$. Note that $M_{k}=U^{\mathrm{H}} S_{k}$ by definition. Using the $N$-point trapezoidal rule, we obtain the following approximation for $S_{k}$ :

$$
\hat{S}_{k}:=\frac{1}{N} \sum_{j=0}^{N-1}\left(\frac{\omega_{j}-\gamma}{\rho}\right)^{k+1} F\left(\omega_{j}\right)^{-1} V, \quad k=0,1, \ldots
$$

The algorithm for the block SS method is shown below.

\section{Algorithm of the block SS method}

Input: $U, V \in \mathbb{C}^{n \times L}, N, K, L, \delta, \gamma, \rho$

Output: $\hat{\lambda}_{1}, \ldots, \hat{\lambda}_{m^{\prime}}, \hat{\boldsymbol{x}}_{1}, \ldots, \hat{\boldsymbol{x}}_{m^{\prime}}$

1. Set $\omega_{j} \leftarrow \gamma+\rho \exp (2 \pi \mathrm{i}(j+1 / 2) / N), j=0,1, \ldots, N-1$

2. Compute $F\left(\omega_{j}\right)^{-1} V, j=0,1, \ldots, N-1$

3. Compute $\hat{S}_{k}, k=0, \ldots, 2 K-1$ by (10)

4. Form $\hat{M}_{k}=U^{\mathrm{H}} \hat{S}_{k}, k=0,1, \ldots, 2 K-1$

5. Construct $\hat{H}_{K L}$ and $\hat{H}_{K L}^{<} \in \mathbb{C}^{K L \times K L}$

6. Perform a singular value decomposition of $\hat{H}_{K L}$

7. Omit small singular value components $\sigma_{j}<\delta \cdot \max _{i} \sigma_{i}$ so that

$\hat{H}_{m^{\prime}}=\hat{H}_{K L}\left(1: m^{\prime}, 1: m^{\prime}\right), \hat{H}_{m^{\prime}}^{<}=\hat{H}_{K L}^{<}\left(1: m^{\prime}, 1: m^{\prime}\right)$, where $m^{\prime} \leq K L$

8. Compute the eigenpairs $\left(\zeta_{1}, \boldsymbol{w}_{1}\right), \ldots,\left(\zeta_{m^{\prime}}, \boldsymbol{w}_{m^{\prime}}\right)$ of the pencil $\hat{H}_{m^{\prime}}^{<}-\lambda \hat{H}_{m^{\prime}}$

9. Construct $S=\left(\hat{S}_{0} \ldots \hat{S}_{m^{\prime}-1}\right)$

10. Compute $\hat{\boldsymbol{x}}_{j}=S \boldsymbol{w}_{j}, \quad j=1,2, \ldots, m^{\prime}$

11. Set $\hat{\lambda}_{j} \leftarrow \gamma+\rho \zeta_{j}, \quad j=1,2, \ldots, m^{\prime}$

In practice, we assign random matrices to $U$ and $V$. We can obtain the eigenvectors corresponding to the eigenvalues whose algebraic multiplicity is less than $L$ by the proposed method.

\section{Numerical Examples}

In this section, we confirm the validity of the proposed method using some nonlinear eigenvalue problems. The algorithm was implemented in MATLAB 7.4. We generated a matrix $V:=\left(\boldsymbol{v}_{1} \ldots \boldsymbol{v}_{L}\right)$ using the MATLAB function rand and set $U=V$. The MATLAB command mldivide was used to evaluate $F(z)^{-1} V$ numerically. The evaluated eigenvectors are normalized so that $\|\hat{\boldsymbol{x}}\|_{2}=1$.

Example 1 We consider the NEP with $F(z)$ that 
Table 1. Relative errors and residuals for Example 1

\begin{tabular}{ccll}
\hline$k$ & $\hat{\lambda}_{k}$ & $\left|\hat{\lambda}_{k}-\lambda_{k}\right|$ & $\frac{\left\|F\left(\hat{\lambda}_{k}\right) \hat{\boldsymbol{x}}_{k}\right\|_{2}}{\left\|F\left(\hat{\lambda}_{k}\right)\right\|_{2}\left\|\hat{\boldsymbol{x}}_{k}\right\|_{2}}$ \\
\hline 1 & -3.141592653589789 & $4.00 \times 10^{-15}$ & $2.58 \times 10^{-12}$ \\
2 & -1.570796326794277 & $6.20 \times 10^{-13}$ & $1.67 \times 10^{-12}$ \\
3 & 0.000000000000661 & $6.61 \times 10^{-13}$ & $1.52 \times 10^{-11}$ \\
4 & 1.570796326761298 & $3.36 \times 10^{-11}$ & $1.11 \times 10^{-10}$ \\
5 & 1.945910151338245 & $2.28 \times 10^{-9}$ & $3.11 \times 10^{-8}$ \\
6 & 3.141592653589055 & $7.39 \times 10^{-13}$ & $3.57 \times 10^{-11}$ \\
\hline
\end{tabular}

Table 2. Residuals for Example 2.

\begin{tabular}{ccc}
\hline$k$ & $\hat{\lambda}_{k}^{1 / 2}$ & $\frac{\left\|F\left(\hat{\lambda}_{k}\right) \hat{\boldsymbol{x}}_{k}\right\|_{F}}{\left\|F\left(\hat{\lambda}_{k}\right)\right\|_{F}\left\|\hat{\boldsymbol{x}}_{k}\right\|_{F}}$ \\
\hline 1 & $0.059793132432759+0.000000862974322 \mathrm{i}$ & $1.41 \times 10^{-15}$ \\
2 & $0.083768827897551+0.000019602073839 \mathrm{i}$ & $6.38 \times 10^{-17}$ \\
3 & $0.084151690319656+0.000003399562592 \mathrm{i}$ & $1.25 \times 10^{-16}$ \\
4 & $0.087765211962668+0.000038185170188 \mathrm{i}$ & $3.47 \times 10^{-17}$ \\
5 & $0.088352686155210+0.000005726087041 \mathrm{i}$ & $3.13 \times 10^{-17}$ \\
6 & $0.093424713463988+0.000393486671297 \mathrm{i}$ & $5.55 \times 10^{-17}$ \\
\hline
\end{tabular}

was transformed using elementary transformations from $\operatorname{diag}\left(\cos (z), \sin (z), \mathrm{e}^{z}-7\right)$. The following list shows the elements of $F(z)$.

$$
\begin{array}{cc}
(1,1) & 2 \mathrm{e}^{z}+\cos (z)-14 \\
(1,2) & \left(z^{2}-1\right) \sin (z)+\left(2 \mathrm{e}^{z}+14\right) \cos (z) \\
(1,3) & 2 \mathrm{e}^{z}-14 \\
(2,1) & (z+3)\left(\mathrm{e}^{z}-7\right) \\
(2,2) & \sin (z)+(z+3)\left(\mathrm{e}^{z}-7\right) \cos (z) \\
(2,3) & (z+3)\left(\mathrm{e}^{z}-7\right) \\
(3,1) & \mathrm{e}^{z}-7 \\
(3,2) & \left(\mathrm{e}^{z}-7\right) \cos (z) \\
(3,3) & \mathrm{e}^{z}-7
\end{array}
$$

The integral path $\Gamma$ taken was as follows:

$$
\Gamma=\gamma+\rho \mathrm{e}^{\mathrm{i} \theta} \quad(\gamma=0, \rho=3.2) .
$$

There are six eigenvalues $\lambda_{1}=-\pi, \lambda_{2}=-\pi / 2, \lambda_{3}=$ $0, \lambda_{4}=\pi / 2, \lambda_{5}=\log 7(\approx 1.9459), \lambda_{6}=\pi$ in $\Gamma$. We took $N=64, K=8, L=2$, and $\delta=10^{-12}$.

The numerical results are shown in Table 1 . We compared the eigenvalues $\left\{\hat{\lambda}_{j}\right\}$ that are obtained by the block SS method to the exact eigenvalues $\left\{\lambda_{j}\right\}$. As shown in Table 1, we obtained all of the eigenvalues in $\Gamma$.

Example 2 We consider the problem that models a radio-frequency gun cavity given in [1] with

$$
F(\lambda)=A_{0}-\lambda A_{1}+i \sqrt{\lambda-\sigma_{1}^{2}} W_{1}+i \sqrt{\lambda-\sigma_{2}^{2}} W_{2},
$$

where $A_{0}, A_{1}, W_{1}, W_{2} \in \mathbb{R}^{9956 \times 9956}$. We took $\sigma_{1}=0$ and $\sigma_{2}=0.043551$. The integral path $\Gamma$ taken was as follows:

$$
\Gamma=\gamma+\rho \mathrm{e}^{\mathrm{i} \theta} \quad(\gamma=0.00625, \rho=0.00375) .
$$

We took $N=64, K=8, L=24$, and $\delta=10^{-12}$.

The numerical results are shown in Table 2. We used Frobenius norm instead of 2-norm. Table 2 shows that the proposed method found six eigenvalues in $\Gamma$. The largest residual of the computed eigenpairs was $1.41 \times 10^{-15}$.

Example 3 Lastly, we consider the problem derived by the delay-differential equation with a single delay
Table 3. Residuals for Example 3.

\begin{tabular}{rrr}
\hline$k$ & $\hat{\lambda}_{k}$ & $\frac{\left\|F\left(\hat{\lambda}_{k}\right) \hat{\boldsymbol{x}}_{k}\right\|_{2}}{\left\|F\left(\hat{\lambda}_{k}\right)\right\|_{2}\left\|\hat{\boldsymbol{x}}_{k}\right\|_{2}}$ \\
\hline 1 & 17.773906360548423 & $2.41 \times 10^{-16}$ \\
2 & 14.471490519110109 & $2.14 \times 10^{-16}$ \\
3 & 8.961335387916407 & $3.43 \times 10^{-16}$ \\
4 & 0.941336550782964 & $1.43 \times 10^{-15}$ \\
5 & -10.407305274429442 & $1.08 \times 10^{-15}$ \\
6 & -31.755615500815374 & $9.43 \times 10^{-16}$ \\
\hline
\end{tabular}

given in [2]:

$$
F(\lambda)=-\lambda I+A_{0}+A_{1} \mathrm{e}^{-\tau \lambda},
$$

where $A_{0}, A_{1} \in \mathbb{R}^{1000 \times 1000}$ are tridiagonal matrices and $I$ is the identity matrix. We took $\tau=0.05$. The integral path $\Gamma$ taken was as follows:

$$
\Gamma=\gamma+\rho \mathrm{e}^{\mathrm{i} \theta} \quad(\gamma=-10, \rho=30) .
$$

We took $N=48, K=16, L=4$ and $\delta=10^{-12}$. It is known that a total of six real eigenvalues lie in $[-40,20]$.

The numerical results are shown in Table 3. As shown in Table 3, the proposed method found all eigenvalues in the specified domain. The largest residual of the computed eigenpairs was $1.43 \times 10^{-15}$.

\section{Conclusion}

In the present paper, we have proposed a numerical method using contour integrals for nonlinear eigenvalue problems of analytic matrix functions. The method is considered as an extension of the numerical method for polynomial eigenvalue problems proposed in [4]. The method enables us to obtain the eigenpairs of analytic matrix functions by solving the generalized eigenvalue problem, which is derived by solving systems of linear equations. Since these linear systems are independent of each other, they can be solved in parallel. In addition, the proposed method does not need fixed point iterations such as Newton's iteration. Error analysis for the proposed method and the estimation of suitable parameters remain as topics for future research.

\section{References}

[1] B. Liao, Subspace projection methods for model order reduction and nonlinear eigenvalue computation, $\mathrm{PhD}$ thesis, Department of mathematics, Univ. of California at Davis, 2007.

[2] E. Jarlebring, The spectrum of delay-differential equations: numerical methods, stability and perturbation, PhD thesis, Inst. Comp. Math, TU Braunschweig, 2008.

[3] T. Sakurai and H. Sugiura, A projection method for generalized eigenvalue problems, J. Comput. Appl. Math., 159 (2003) 119-128.

[4] J. Asakura, T. Sakurai, H. Tadano, T. Ikegami and K. Kimura, A numerical method for polynomial eigenvalue problems using contour integral, submitted.

[5] I. Gohberg and L. Rodman, Analytic matrix functions with prescribed local data, J. d'Analyse Mathématique, 40 (1981) 90-128.

[6] T. Ikegami, T. Sakurai, and U. Nagashima, A filter diagonalization for generalized eigenvalue problems based on the Sakurai-Sugiura method, Tech. Rep. CS-TR-08-13, Univ. of Tsukuba, 2008. 